\title{
MAXIMIZE THE LIFETIME OF SENSOR NETWORK BY LOAD BALANCING USING TREE TOPOLOGY
}

\author{
S.S. Patil and B.S. Shetty \\ Department of Information Technology, Walchand College of Engineering, India
}

\begin{abstract}
In many wireless sensor networks due to the limited energy of sensor nodes energy conservation is one of the most important challenges. To enhance the lifetime of the network emphasis is given to design energy efficient routing algorithms. In WSN, sensor nodes which are nearer to the base station having a task of collecting data for the entire area and send to the base station. This node has an additional load and depletes its energy faster. This paper addresses the problem of lifetime maximization by load balancing. This paper proposes energy efficient load balanced data collection algorithm considering different network parameter (e.g., density, degree). In this method, Data collection tree topology is built at the sink node. Performance of the proposed algorithm is evaluated by considering various parameters like topology, availability of resources and the energy utilization of nodes in different paths of the tree, which may vary and ultimately impacts the overall network lifetime. Sensor nodes are switched from their original path to other based on the load and it reduces communication overhead.
\end{abstract}

Keywords:

Load Balancing, Energy Efficiency, Data Collection Tree, Convergence Time

\section{INTRODUCTION}

A Wireless Sensor Network (WSN) is a wireless network which consists of spatially distributed sensor nodes that are used in a variety of applications like environmental monitoring and surveillance. These sensor nodes are worked in a cooperative manner with each other and they communicate wirelessly. Sensor nodes are limited in battery-powered, computation capability and memory [1]. Sensor nodes are randomly deployed over the sensing area or monitoring area. In WSN, typically data are gathered from all the sensor nodes and submitted to the point called a base station or sink node. Thus, all the sensor nodes are arranged in tree topology [2] which is grounded at the sink. Sensor nodes are severely battery-limited [3]. Sensor nodes are run on battery power and that is not replaceable. So energy saving [4] is an extensive challenge in a sensor network. In previous work, a lot of research has been done on energy preservation. Different techniques are used for the energy preservation in sensor network such as dutycycling [3], aggregation of data [4], and balancing energy level [5][6]. In this paper, load balancing approach is used to find the duration maximization problem [7] [8]. Mainly focus on the level of aggregation to maximize the lifetime of the network. Yet existing approaches in this area resides big challenges such as a situation where topology changes repeatedly and in terms of time complexity. Load balancing approach is used to distribute the load as uniform as possible over the region. Assume that all the sensor nodes are densely deployed over the region. Select any random tree [9] from the initial connectivity graph. Then tried to achieve balanced tree structure by considering the degree of the node. In the literature, the lifetime of the network has been measured in different ways [10]. Work addresses lifetime maximization of tree topology and considers the duration is the time until the initial node in the region finishes [6] all of its energy. This definition is application-independent and, thus, is suitable for varied scenarios. In this paper, majorly focus on the level of aggregation over the tree structure and send only aggregated data to the base station. Achieved balanced tree structure supports full aggregation because a load of sensor nodes depends on the degree of the node. The key distributions of this paper are as follows. First, classify various energy efficient algorithms for the balancing load. Second, find out the duration maximization problem of the tree structure in a sensor network. Third, constructed balanced tree structure using the proposed algorithm.

\section{RELATED WORK}

In recent years, energy-efficient routing communication has become major challenges. The following survey provides the basis for proposed work. Many solutions are proposed in the literature for load balancing in a sensor network. A node-centric approach is used to maximize the network lifetime [11]. Their load balancing scheme increases trees repeatedly from the sink. This algorithm presents an explanation for forming an initial tree and rebalances the tree using topological knowledge instead of randomly. They focused on wireless-sensor-network with an asymmetric architecture and considered centralized architecture only. Also, assume the common case in which position of the sensor node is fixed. The work proposed in [12] for constructing maximum lifetime of data gathering tree in a sensor network. They have provided an optimal solution within the solvable period by investing its structure. It gives outcomes in better approximation ratio to extend the duration of network and also reduces the load of the base station. They have been implemented decentralized approach among trees but still in local-tree sink node make centralized-decisions. The local wiser algorithm proposed in [13] for maximizing network lifetime using load balancing. They proposed the network-lifetime-maximization problem which maps with "localized-cost-balancing" problem. The following characteristics are provided by given approach: computing locally, stable and provides an optimal solution to extend network-lifetime, easily-implemented and self-adaptive. The work in [14] proposed for maximizing the lifetime of shortest path tree within polynomial time. For solving minimum spanning tree problem they used load-balancing scheme. Here, they considered aggregation in the network at every level of fat-tree and applies the "min-cost-max-flow" approach. Energy efficient algorithm is demonstrated in [15] for distributing the load over the network using randomized changing (switching). They extend the lifetime of data gathering tree in WSN by applying load balanced scheme. Based on the concept of "bounded-balanced-trees" this algorithm randomly switches the data forwarding-paths of nodes 
i.e. randomly switches some sensor nodes from their original path to another path. In this paper, authors have been proved that proposed algorithm requires less complexity to covered deployment area also it has fast convergence time related to existing MIIT, LOCAL-OPT algorithm. According to this strategy as iterations proceed nodes progressively have a lower probability of the changing path. To reduce this drawback of the existing algorithm this paper focusing on the degree of node parameter for improving the performance of the algorithm. This paper proposes an Energy efficient load balanced data collection algorithm using balanced tree concepts to extend the lifetime of the network. The dense tree is chosen considering the degree of the node instead of the random tree as an input from network connectivity graph. The algorithm is terminated until the balanced tree [16] is found i.e. all the sensor nodes are having a uniform load. The major contribution of this paper is that focusing on to extend the lifetime of data collection tree and to reduce communication overhead. Existing approaches are not suitable for changing the path as iterations proceed also it requires high communication overhead to find out descendants node for changing the path. In contrast, choose the arbitrary tree. It supports for full aggregation because the size of subtree depends on the degree of the node so ultimate it extends the lifetime of data collection tree by reducing raw data. The probability of selecting descendent nodes is more for changing the path. The definition of a lifetime of the network is different in the literature survey. Network lifetime has measured until the first node finishes all of its energy. Many solutions proposed in the literature for the extending the network lifetime. The work defined in the literature in terms of coverage [17], which is application-specific and assume that the location of sensor nodes is known [18] and do not characterize the convergence. In some scenarios, only local information is used. In across, take network-oriented approaches and do not rely on the location of sensor nodes. This paper proposes energy efficient load balanced data collection algorithm for maximizing the lifetime of the network by considering different parameters of the network in order to reduce the communication overhead.

\section{PROBLEM FORMULATION}

The problem of lifetime maximization for the data collection tree in a sensor network is defined using the concept of a balanced tree.

\subsection{NETWORK MODEL}

The network is a connected graph which contains $G=\{V, E\}$, where $V$ is the set of vertices corresponding to the $N$ sensor nodes and $E$ is the edges between nodes. Tree topology is used for deployment of $N$ sensor nodes over the network. The root node acts as a base station or sink node which collects all the sensed information from all the nodes over the network.

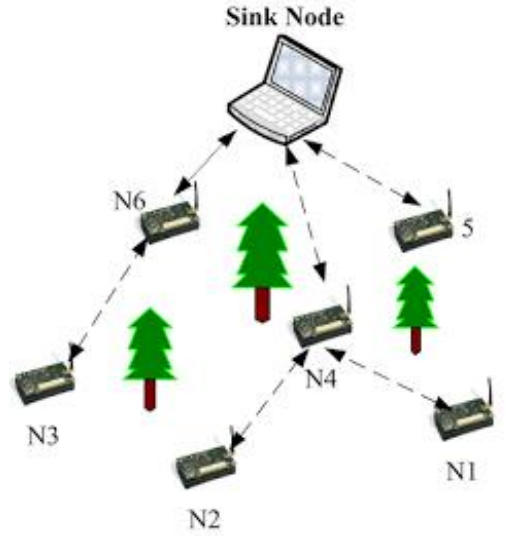

Fig.1. Tree topology in a sensor network

Data collection tree is a common operation in a sensor network in which data is collected from all the sensor nodes over the network. This collection of data is sent towards the common sink over tree-based topology. Fast data collection tree [19] is made using full aggregation instead of raw data. Sink collects all the data from the network. This process is called as data collection round [20]. In this proposed algorithm assumption are made that sensor nodes are having different initial energy resources.

Following characteristics are assumed according to the [6]:

- All the nodes have different initial energy and sink node has an infinite energy with powerful computation ability.

- Each node within the network has same communication range and requires multi-hop communication for transmitting the data towards the sink.

- Nodes basically consume energy in the communication

The amount of data is received from children in each data collection round is assumed to be $R_{i}^{c}$ whereas $R_{i}{ }^{t}$ is the amount of data transmitted towards children from its parent. The units for data transmission and reception are assumed $E^{t}$ and $E^{c}$ respectively. From the above assumption, calculate energy loss rate $r_{i=} R_{i}^{t} E^{t}+R_{i}^{c} E^{c}$. A load of a node is the ratio of energy loss rate to the energy budget i.e. $\gamma=r_{i} / e_{i}$. The lifetime of a node is defined as the ratio of current energy budget to the energy loss rate i.e. $t_{i}$ $=e_{i} / r_{i}$.

\section{PROPOSED METHODOLOGY}

This section presents an Energy efficient load balanced data collection algorithm for maximizing the lifetime of wireless sensor network considering load balancing scheme. This algorithm searches bottleneck node and balances path loads of those nodes in the network through randomized changing the path and transforming the tree into a balanced tree. The basic idea of this algorithm is to generate energy-efficient balanced tree by changing the path from original to other with lower load, considering the threshold value. In sensor network after certain iterations found that some sensor nodes are going to die. In that case, the algorithm searches the node (bottleneck) which depletes its energy earlier. So mainly focusing on to distribute the load as uniform as possible throughout the network. Implementation is done with the dense network. According to topological information choose a dense tree and checked condition for a balanced tree. If the difference between maximum path load and 
minimum path load is greater than the threshold value then given define tree is not balanced. Data collection trees are built from connectivity graph and applying different steps of the algorithm which goes through series of iterations until a balanced tree is found. This algorithm is terminated when the balanced tree is found or maximum time an individual node is selected for changing the path. The Fig.2 shows the flow of an Energy efficient load balanced data collection algorithm. First, initialized the node load and path load of all the nodes in the defined tree and set initially probability for every node is 0.5 for switching or changing the path and count is 0 .

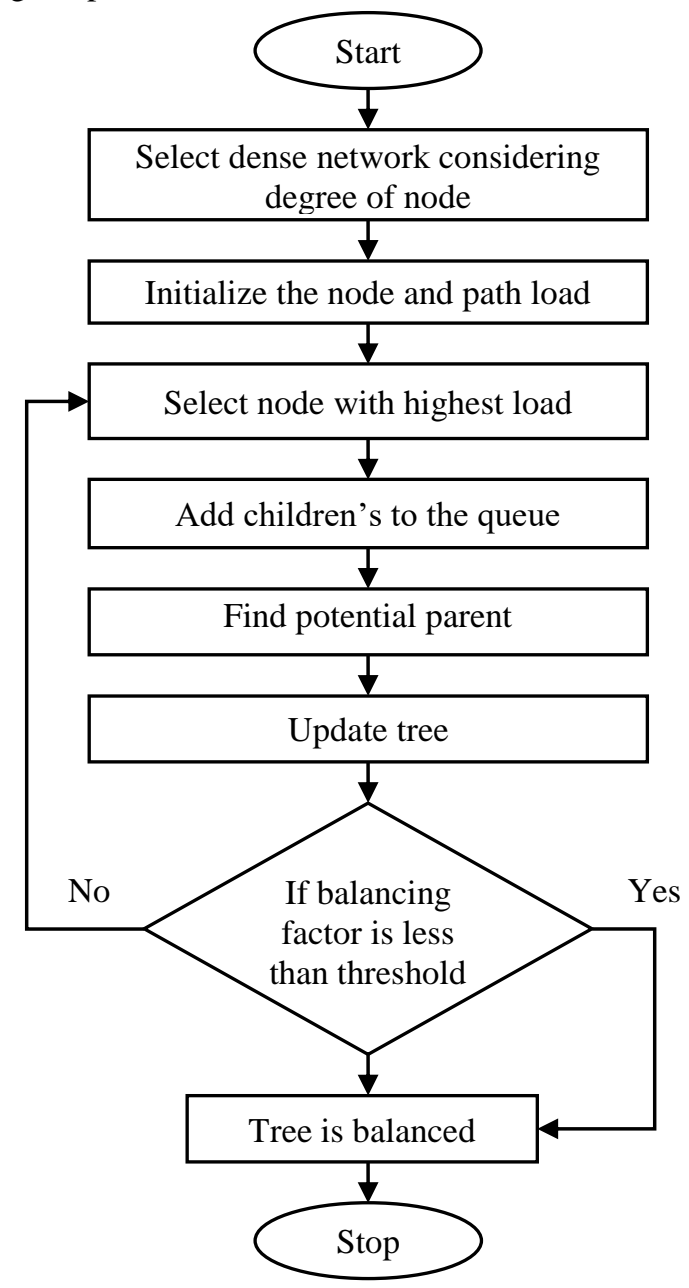

Fig.2. Flowchart of the proposed algorithm

This count is incremented when an individual node is selected for switching repeatedly. Then switch function find out the load of the defined tree and choose such a node which is having a high load. Then children's are added to the list for changing the path Select one node randomly from that list by considering potential edges (the edges can be used for changing the path in the next iteration). After that, algorithm search potential parent for that selected node using potential parent function. Then check all the neighbors of that selected node from the list and pick such a node from neighboring nodes which is having fewer paths than selected node and more than the threshold (i.e. load balancing parameter). Finally, update all the node load and path load after every changing the path.

\section{RESULT AND DISCUSSION}

This section evaluates the performance of the proposed algorithm in MATLAB. First, initialized the node load and path load of all the nodes in the defined tree and set initially the probability for every node is 0.5 for changing the path and count is 0 . This count is incremented when an individual node is selected for changing repeatedly. Then switch function find out the load of the defined tree and choose bottleneck node. Then children's are added to the list for changing the path. The algorithm searches potential parent for that selected node using potential parent function. Then check all the neighbors of that selected node from the list and pick such a node from neighboring nodes which is having less path load than selected node and more than the threshold. Update all the node load and path load after every round. The Fig.3 shows the lifetime achieved by the steady state of the network. The threshold value is the difference between maximum path load and minimum path load in given tree. Constructed different programs for varying different sensor nodes and the threshold value and find out the optimal value of threshold value.

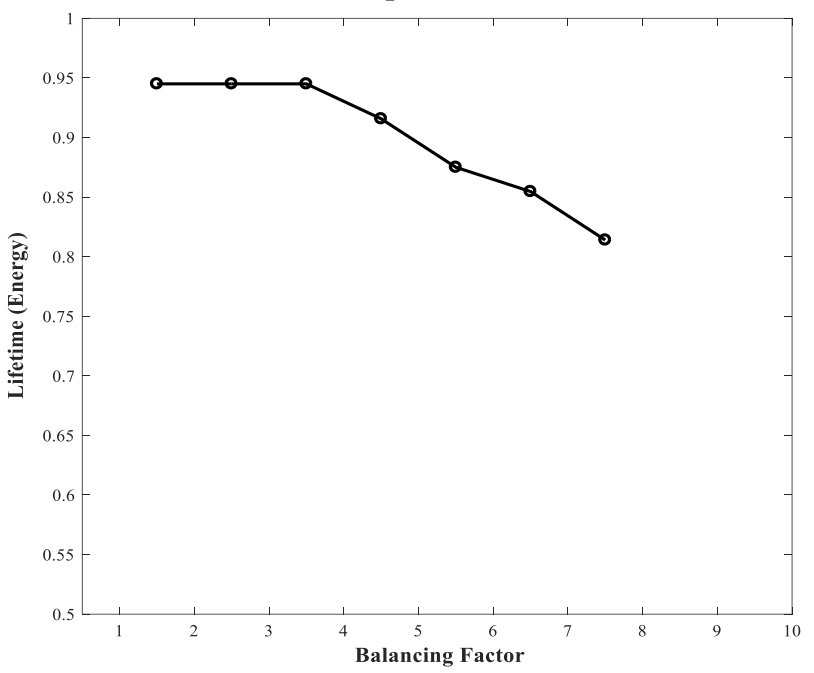

Fig.3. Impact of balancing factor on lifetime when the threshold varies from 1 to 10

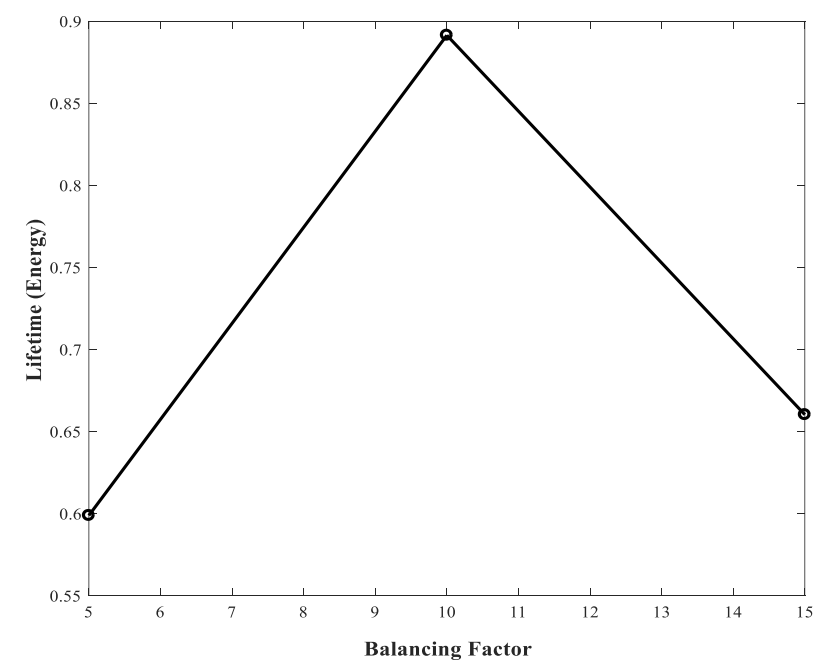

Fig.4. Impact of balancing factor on lifetime when the threshold varies from 5 to 15 
The Fig. 3 and Fig.4 shows that when balancing factor is less than the lifetime of the network is increased. In oppose lifetime of the network is decreases when balancing factor is more. Constructed different graphs varying multiple sensor nodes and the threshold value. In Fig. 3 thresholds is varying from 1 to 10 but this low value is not possible in reality. According to experiments proposed algorithm achieved better results at steady state when the threshold value is in between 5 to 10 . According to this strategy as iterations proceed nodes progressively have a lower probability of changing the path. To overcome this drawback of the existing algorithm this paper focuses on the degree of node parameter for improving the performance of the algorithm.

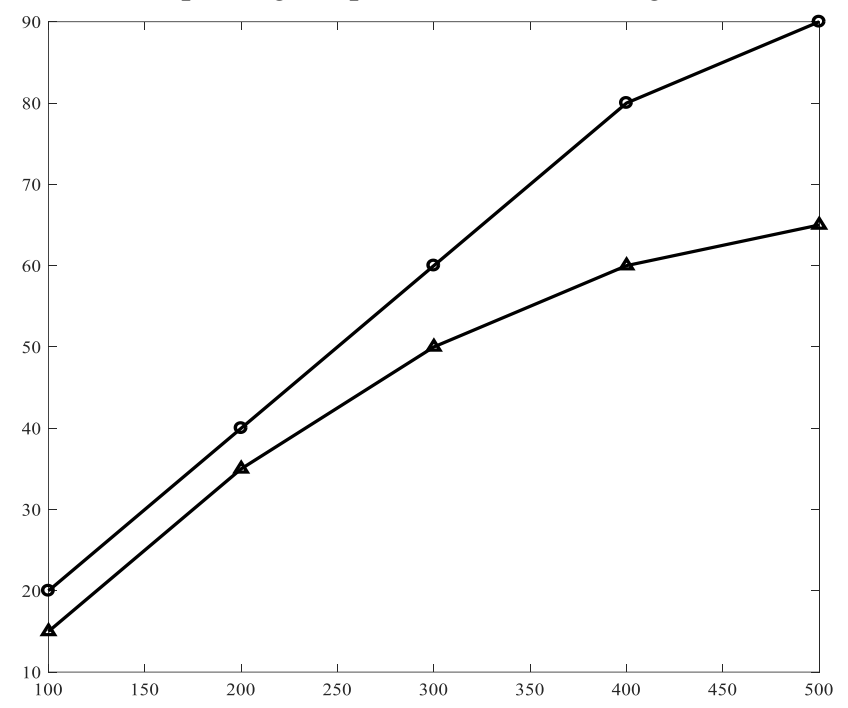

Fig.5. Average numbers of iterations for changing the path

The Fig. 5 shows the average number of iterations to obtain the balanced tree using the proposed algorithm. In sparse tree network numbers of iterations are more to search potential parent and changing the path of sensor nodes from original to other for getting a balanced tree. It has more burdens on the network. In a dense tree network, fewer numbers of iterations are required to get a balanced tree because a number of potential parents are available for the bottleneck node for distributing the load over the network. Communication overhead decreases as compared to the sparse network. The sparse network is not suitable in case of full aggregation where the as dense network is better for full aggregation because a load of sensor nodes depends on the degree of the node. The Fig. 6 shows the impact of aggregation on a lifetime of the network. When full aggregation is used, proposed energy efficient algorithm obtains better lifetime with respect to the existing algorithm and it requires less communication overhead. Performance of the proposed algorithm is evaluated by varing a number of nodes and level of aggregation. The value ' 0 ' represents no aggregation whereas ' 1 ' represents full aggregation i.e. combined all the data.

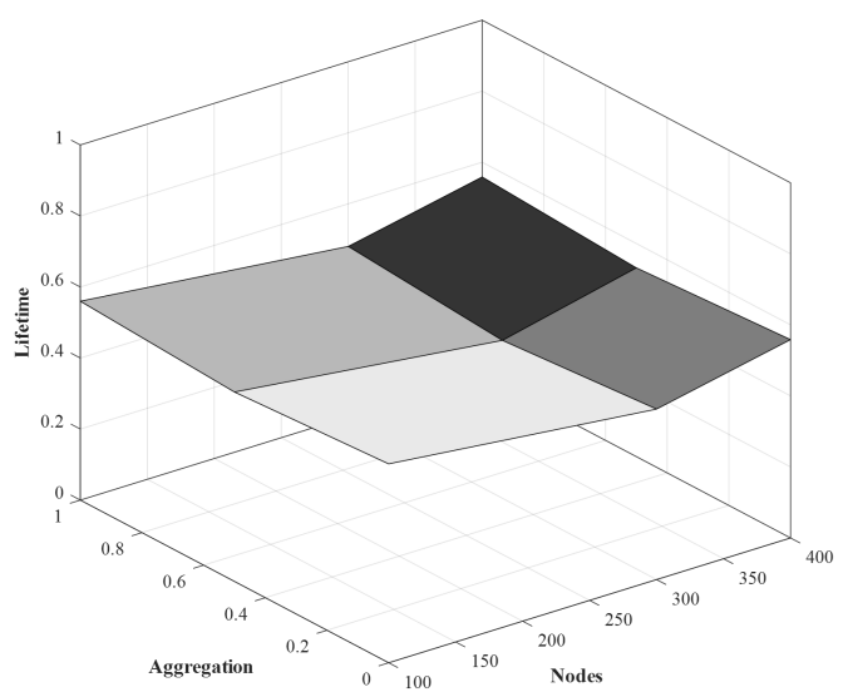

Fig.6. Impact of aggregation on lifetime

Here dense network is chosen with considering the degree of node parameter to reduce communication delay in the network. A load of the node depends on the degree of the node rather than the size of the subtree. From the above discussion, it is clear that proposed Energy efficient load balanced data collection algorithm gives better performance when the level of aggregation is increased. For large scale network, it requires less time complexity in terms of a number of iterations with respect to the existing algorithm. Communication delay is minimizing with respect to existing $\mathrm{n} \mathrm{m}$; approaches. It gives better performance for the dense network. Fast data collection tree is achieved using full aggregation in tree-based topology.

\section{CONCLUSION AND FUTURE SCOPE}

Numerous researchers have been proposed in the literature for maximizing the network lifetime. Different work is defined in the literature considering coverage factor, but that is useful only for specific application. In this existing approach as iterations proceed nodes progressively have a lower probability of neural networks. In ongoing research, this paper focuses on tree topology to reduce communication overhead with considering coverage area.

\section{REFERENCES}

[1] I.F. Akyildiz, W. Su, Y. Sankarasubramaniam and E. Cayirci, "Wireless Sensor Networks: A Survey", Computer Networks, Vol. 38, No. 4, pp. 393-422, 2002.

[2] G. Anastasi, M. Conti, M. Di Francesco and A. Passarella, "Energy Conservation in Wireless Sensor Networks: A Survey", Ad Hoc Networks, Vol. 7, No. 3, pp. 537-568, 2009.

[3] G. A1nastasi, M. Conti and M. Di Francesco, "Extending the Lifetime of Wireless Sensor Network through Adaptive Sleep", IEEE Transactions on Industrial Informatics, Vol. 5, No. 3, pp. 351-365, 2009.

[4] Antoni Morell et al., "Data Aggregation and Principal Component Analysis in WSNs", IEEE Transactions on Wireless Communications, Vol. 15, No. 6, pp. 3908-3919, 2015. 
[5] S. Toumpis and S. Gitzenis, "Load Balancing in Wireless Sensor Networks using Kirchhoff's Voltage Law", Proceedings of IEEE INFOCOM, pp. 1656-1664, 2009.

[6] J. Liang, J. Wang, J. Cao, J. Chen and M. Lu, “An Efficient Algorithm for Constructing Maximum lifetime Tree for Data Gathering Without Aggregation in Wireless Sensor Networks", Proceedings of IEEE INFOCOM, pp. 1-5, 2010.

[7] Y. Liu, Y. He, M. Le, J. Wang, K. Liu, and L. Mo, "Does Wireless Sensor Network Scale? A Measurement Study on Green Orbs", IEEE Transactions on Parallel and Distributed Systems, Vol. 24, No. 10, pp. 873-881, 2011.

[8] A. Willig, "Recent and Emerging Topics in Wireless Industrial Communications: A Selection", IEEE Transactions on Industrial Informatics, Vol. 4, No. 2, pp. 102-124, 2008.

[9] Debraj De and Sajal K. Das, "SREE-Tree: SelfReorganizing Energy-Efficient Tree Topology Management in Sensor Networks", Proceedings of International Conference on Sustainable Internet and ICT for Sustainability, pp. 113-119, 2015.

[10] Yunxia Chen and Qing Zhao, "On the Lifetime of Wireless Sensor Networks", IEEE Communications Letters, Vol. 9, No. 11, pp. 976-978, 2005.

[11] H. Dai and R. Han, "A Node-Centric Load Balancing Algorithm for Wireless Sensor Networks", Proceedings of IEEE Global Telecommunications Conference, pp. 548-552, 2003.

[12] Y. Wu, Z. Mao, S. Fahmy, S. Member and N.B. Shroff, "Constructing Maximum-Lifetime Data-Gathering Forests in Sensor Networks", IEEE/ACM Transactions on Networking, Vol. 18, No. 5, pp. 1571-1584, 2010.

[13] Y. Wang, Y. Wang, H. Tan and F.C.M. Lau, "Maximizing Network Lifetime Online by Localized Probabilistic Load
Balancing", Proceedings of International Conference on Ad-hoc, Mobile, and Wireless Networks, pp. 332-345, 2011.

[14] D. Luo, X. Zhu, X. Wu and G. Chen, "Maximizing Lifetime for the Shortest Path Aggregation Tree in Wireless Sensor Networks", Proceedings of IEEE INFOCOM, pp. 15661574, 2011.

[15] S.K.A. Imon, A. Khan, M. Di Francesco and S.K. Das, "Energy-Efficient Randomized Switching for Maximizing Lifetime in Tree-based Wireless Sensor Networks", IEEE/ACM Transactions on Networking, Vol. 23, No. 5, pp. 1401-1415, 2015.

[16] U. Monaco, F. Cuomo, T. Melodia, F. Ricciato and M. Borghini, "Understanding Optimal Data Gathering in the Energy and Latency Domains of a Wireless Sensor Network", Computer Networks, Vol. 50, No. 18, pp. 3564 3584, 2006.

[17] Q. Zhao and M. Gurusamy, "Lifetime Maximization for Connected Target Coverage in Wireless Sensor Networks", IEEE/ACM Transactions on Networking, Vol. 16, No. 6, pp. 1378-1391, 2008.

[18] Z. Wang, E. Bulut and B. Szymanski, "Energy Efficient Collision Aware Multipath Routing for Wireless Sensor Networks", Proceedings of IEEE International Conference on Communications, pp. 1-5, 2009.

[19] Ozlem Durmaz Incel, Amitabha Ghosh, Bhaskar Krishnamachari and Krishnakant Chintalapudi, "Fast Data Collection in Tree-Based Wireless Sensor Networks", IEEE Transactions on Mobile Computing, Vol. 11, No. 1, pp. 8699, 2012.

[20] H.O. Tan and I. Korpeoglu, "Power Efficient data Gathering and Aggregation in Wireless Sensor Networks", ACM SIGMOD Record, Vol. 32, No. 4, pp. 66-71, 2003. 